\begin{tabular}{|c|c|}
\hline 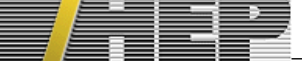 & PUBLISHED FOR SISSA BY SPRINGER \\
\hline & $\begin{array}{r}\text { ReCEIVEd: May 1, } 2018 \\
\text { ACCEPtED: June 5, } 2018 \\
\text { Published: June 12, } 2018\end{array}$ \\
\hline
\end{tabular}

\title{
Volume and complexity for Warped AdS black holes
}

\author{
Roberto Auzzi, ${ }^{a, b}$ Stefano Baiguera ${ }^{c}$ and Giuseppe Nardelli ${ }^{a, d}$ \\ ${ }^{a}$ Dipartimento di Matematica e Fisica, Università Cattolica del Sacro Cuore, \\ Via Musei 41, 25121 Brescia, Italy \\ ${ }^{b}$ INFN Sezione di Perugia, Via A. Pascoli, 06123 Perugia, Italy \\ ${ }^{c}$ Università degli studi di Milano Bicocca and INFN, Sezione di Milano - Bicocca, \\ Piazza della Scienza 3, 20161, Milano, Italy \\ ${ }^{d}$ TIFPA - INFN, c/o Dipartimento di Fisica, Università di Trento, \\ 38123 Povo (TN), Italy \\ E-mail: roberto.auzzi@unicatt.it, s.baiguera@campus.unimib.it, \\ giuseppe.nardelli@unicatt.it
}

ABSTRACT: We study the Complexity=Volume conjecture for Warped $\mathrm{AdS}_{3}$ black holes. We compute the spatial volume of the Einstein-Rosen bridge and we find that its growth rate is proportional to the Hawking temperature times the Bekenstein-Hawking entropy. This is consistent with expectations about computational complexity in the boundary theory.

KEYWORDS: AdS-CFT Correspondence, Black Holes

ARXiv EPrint: 1804.07521 


\section{Contents}

1 Introduction 1

2 Black holes in Warped AdS 3

2.1 Conserved charges and thermodynamics 4

2.2 Expectations for the asymptotic rate of growth of complexity 5

2.3 Eddington-Finkelstein coordinates 6

3 Complexity=volume $\quad 7$

3.1 Einstein-Rosen Bridge $\quad 7$

$\begin{array}{lll}3.2 & \text { Non-rotating case } & 7\end{array}$

3.3 Rotating case 9

4 Conclusions $\quad 12$

$\begin{array}{ll}\text { A An explicit model } & 14\end{array}$

\section{Introduction}

Several quantum information concepts have been fruitfully applied to the investigation of fundamental questions in gravity: classical spacetime geometry seems somehow to hiddenly encode information properties of a dual quantum system. Bekenstein-Hawking entropy is indeed proportional to the area of the event horizon [1] and the laws of Black Hole (BH) mechanics have a deep connection with thermodynamics [2]. The microscopic derivation of the BH entropy in string theory given by Strominger and Vafa [3], even if valid just for some particular extremal cases, suggests that the $\mathrm{BH}$ horizon area should be directly linked to the number of microstates in some appropriate dual description.

The AdS/CFT correspondence provides an interesting theoretical laboratory to investigate quantum information in gravity. An example which has given us a lot of interesting insights is the Ryu-Takayanagi construction [4-6]: it links the entanglement entropy of the dual conformal field theory (CFT) to the geometrical area of a bulk minimal surface hanging from the boundary. Entropy should be somehow related to the counting of degrees of freedom in the dual quantum description of a $\mathrm{BH}$. On the other hand, it turns out that entropy is not the correct quantity to focus on in order to understand the growth of the Einstein-Rosen bridge (ERB) in the interior of a $\mathrm{BH}[7,8]$. Indeed, the ERB connecting two boundaries in an eternal AdS black hole continues to grow for a time scale which is much longer compared to the thermalization scale.

Eternal BHs in AdS space are dual to two entangled copies of the same CFT living on each of the boundaries [9]. If we take both the times $t_{L}, t_{R}$ of the left and the right CFTs 
running forward, this geometry is dual to a time-dependent thermofield doublet state [10]. The size of the ERB connecting the left and the right boundary asymptotically grows linearly with time.

A promising candidate to capture this growth in the dual boundary theory is computational complexity $[7,8,11-13]$. For a quantum system, it is defined as the minimal number of elementary unitary operations needed to reach a given quantum state, starting from an initial reference state. In the case of quantum mechanics with a finite number of degrees of freedom, Nielsen and collaborators [14, 15] introduced a nice geometrical tool: the problem of quantum complexity is traced back to finding geodesics in the space of unitary evolutions. In order to extend such analysis in quantum field theories, many subtleties arise and only recently complexity calculations have been carried out for free field theories [16-18]. Another approach to complexity in quantum field theory uses the Liouville action [19] in connection with tensor networks [20].

Two different holographic duals of quantum complexity have been proposed so far: the complexity=volume (CV) conjecture $[7,8,11]$ and the complexity=action (CA) conjecture $[12,13]$. CV relates complexity to the volume of a codimension one surface (with maximal volume) anchored at the boundary:

$$
C_{V} \propto \operatorname{Max}\left(\frac{V}{G l}\right)
$$

where $G$ is the Newton constant and $l$ the AdS radius. CA relates complexity to the gravitational action $I_{\mathrm{WDW}}$ evaluated in a Wheeler-DeWitt patch:

$$
C_{A}=\frac{I_{\mathrm{WDW}}}{\pi} .
$$

Both the conjectures have their own merits. In particular, while CV explicitly depends on the AdS curvature $l$, CA looks more universal, because no explicit factors related to the asymptotic of the space are present. On the other hand, recent works show that CA seems to overshoot the Lloyd's bound [21] at intermediate times [22] and moreover seems to give some curious and weird features: complexity is constant before some initial time $\tau_{c}$ and immediately after this time $\frac{d C_{A}}{d \tau}$ is divergent and negative. On the other hand, CV behaves as a monotonic and smooth function of the time. Another merit of CV conjecture is that it can be naturally extended to consider subregions [23-25]. Another holographic interpretation of the volume was proposed in [26].

Complexity is interesting not only to capture the linear growth of ERBs inside BHs; for example, complexity of formation of $\mathrm{BH}$ was studied in [27]. It is also interesting to study complexity in connection to other spacetimes; for example, the relation between complexity and spacetime singularities was investigated in [28, 29] and complexity for the AdS soliton was studied in [30]. Complexity for quenches and time-dependent couplings was studied in [31, 32]. The effect of dilaton was discussed in [33].

It is interesting to consider extensions of holography to spacetimes that are not asymptotically AdS. The most relevant cases for physical applications would be flat or de Sitter spaces; unfortunately in these cases very little is known about the dual field theory. It is 
then interesting to study non-trivial modifications of AdS/CFT where we have more direct information about the structure of the dual. One of these cases is Warped $\mathrm{AdS}_{3} / \mathrm{CFT}_{2}[34-$ 37], which is a correspondence between gravity in $2+1$ dimensions in spaces with Warped $\mathrm{AdS}_{3}$ (WAdS) asymptotic and a putative Warped Conformal Field Theory (WCFT) in $1+1$ dimensions. In recent times there have been significant progresses in the study of this extension of the AdS/CFT correspondence. Using warped conformal symmetries, an analog of Cardy formula was derived in [35]; in [36] some free examples of WCFT were built. Entanglement entropy in WAdS space and in WCFT was studied by several authors, e.g. [38-40]. In this paper we will address the CV conjecture for BHs in WAdS spaces.

The paper is organized as follows. In section 2 we review the Warped black holes solution and we discuss mass and angular momentum in Einstein gravity; an explicit example in a theory with ghosts is discussed in appendix A. In section 3 we compute the growth rate of the volume of the ERB as a function of time, both for the non-rotating and rotating cases. We conclude in section 4 .

\section{Black holes in Warped AdS}

We will be interested in BHs with $\mathrm{WAdS}_{3}$ asymptotic $[34,41,42]$. This class of metrics should be dual to a boundary WCFT at finite temperature. For the metric we use the notation of [34]:

$$
\begin{aligned}
\frac{d s^{2}}{l^{2}} & =d t^{2}+\frac{d r^{2}}{\left(\nu^{2}+3\right)\left(r-r_{+}\right)\left(r-r_{-}\right)}+\left(2 \nu r-\sqrt{r_{+} r_{-}\left(\nu^{2}+3\right)}\right) d t d \theta+\frac{r}{4} \Psi d \theta^{2}, \\
\Psi(r) & =3\left(\nu^{2}-1\right) r+\left(\nu^{2}+3\right)\left(r_{+}+r_{-}\right)-4 \nu \sqrt{r_{+} r_{-}\left(\nu^{2}+3\right)},
\end{aligned}
$$

where $0 \leq r<\infty,-\infty<t<\infty, \theta \sim \theta+2 \pi$ and the horizons are located at $r=r_{+}, r_{-}$ with $r_{+} \geq r_{-}$. Taking $r_{+}=r_{-}=0$ we obtain the WAdS spacetime in Poincaré patch. The boundary is parameterized by $(\theta, t)$ and the spatial geometry is flat. The parameter $\nu$ is related to the left and right central charges of the boundary WCFT, which in Einstein gravity are [43]

$$
c_{L}=c_{R}=\frac{12 l \nu^{2}}{G\left(\nu^{2}+3\right)^{3 / 2}} .
$$

For $\nu=1$ the Banados-Teitelboim-Zanelli (BTZ) black hole [44, 45] is recovered; in this case, the following change of coordinates

$$
r=\bar{r}^{2}, \quad t=\frac{\sqrt{r_{+}}-\sqrt{r_{-}}}{l^{2}} \bar{t}, \quad \theta=\frac{l \bar{\theta}-\bar{t}}{l^{2}\left(\sqrt{r_{+}}-\sqrt{r_{-}}\right)}, \quad r_{ \pm}=\bar{r}_{ \pm}^{2},
$$

brings the metric to the standard BTZ form:

$$
d s^{2}=-\frac{\bar{r}^{2}-\bar{r}_{+}^{2}-\bar{r}_{-}^{2}}{l^{2}} d \bar{t}^{2}+\frac{l^{2} \bar{r}^{2}}{\left(\bar{r}^{2}-\bar{r}_{+}^{2}\right)\left(\bar{r}^{2}-\bar{r}_{-}^{2}\right)} d \bar{r}^{2}-2 \frac{\bar{r}_{+} \bar{r}_{-}}{l} d \bar{t} d \bar{\theta}+\bar{r}^{2} d \bar{\theta}^{2} .
$$

The case $\nu=1$ admits the $\mathrm{AdS}_{3}$ symmetries, i.e. $\mathrm{SL}(2, \mathbb{R})_{L} \times \mathrm{SL}(2, \mathbb{R})_{R}$, which for generic $\nu$ are broken to the $\mathrm{WAdS}_{3}$ symmetry group, that is $\mathrm{SL}(2, \mathbb{R})_{L} \times \mathrm{U}(1)_{R}$. 
For $\nu^{2}<1$ the solution is pathological because it has closed time-like curves. For $\nu^{2}>1$ the solution is not sick and can be realized as an exact vacuum solution of Topologically Massive Gravity (TMG) [41, 42], New Massive Gravity (NMG) [46] and also general linear combinations of the two mass terms [47]. We restrict our analysis to the case of positive $\nu$. So at the end we will consider just the case $\nu \geq 1$.

Strictly speaking, the relation between area and entropy holds just in Einstein gravity: if we consider higher order corrections to the gravitational entropy, we have to use the Wald entropy formula [48] instead of the geometrical area law. So the CV conjecture should be directly applicable just to Einstein gravity and should be appropriately modified in order to take into account higher order corrections in the gravitational action. A proposal for such correction has been put forward in $[23,49]$. The CA conjecture can also be generalized to the case of higher derivatives corrections to the gravitational action, see e.g. [50-52].

As far as we know, there is no known non-pathological matter content in field theory supporting stretched warped BHs in Einstein gravity [43]. However, they can be obtained as solutions to a perfect fluid stress tensor with spacelike quadrivelocity [53]. Alternatively they can arise as a solution of Chern-Simons-Maxwell electrodynamics coupled to Einstein gravity $[54,55]$, but a wrong sign for the kinetic Maxwell term is required in order to have solutions with no closed time-like curves (which corresponds to $\nu^{2} \geq 1$ ). Moreover, warped $\mathrm{BH}$ can arise in string theory constructions, e.g. [56-58]. In the following we take a pragmatical approach: we suppose that a consistent realization of stretched warped BHs in Einstein gravity exists, and we investigate the CV conjecture.

\subsection{Conserved charges and thermodynamics}

In order to compare with the expectations for complexity, we need to discuss conserved charges and thermodynamical quantities. In the Einstein case, the entropy is given by the area of the outer horizon:

$$
S=S_{+}=\frac{l \pi}{4 G}\left(2 \nu r_{+}-\sqrt{r_{+} r_{-}\left(\nu^{2}+3\right)}\right) .
$$

At least formally, we can also define the entropy associated to the inner horizon:

$$
S_{-}=\frac{l \pi}{4 G}\left(\sqrt{r_{+} r_{-}\left(\nu^{2}+3\right)}-2 \nu r_{-}\right) .
$$

The Hawking temperature and the angular velocity are given by [34]:

$$
T=\frac{\nu^{2}+3}{4 \pi l} \frac{r_{+}-r_{-}}{2 \nu r_{+}-\sqrt{\left(\nu^{2}+3\right) r_{+} r_{-}}}, \quad \Omega=\frac{2}{\left(2 \nu r_{+}-\sqrt{\left(\nu^{2}+3\right) r_{+} r_{-}}\right) l} .
$$

The first law of thermodynamics gives:

$$
d M=T d S+\Omega d J .
$$

Following [59, 60], the existence of a holographic dual implies a quantization condition on the product of inner and outer entropies, which in turn must be proportional to the 
conserved charges of the black hole which are quantized. Since the angular momentum is the only quantized conserved charge, we obtain $J=S_{-} S_{+} f(\nu)$, where $f(\nu)$ is a so far arbitrary function which will be fixed by thermodynamics.

Imposing that the resulting $d M$ is an exact differential, the function $f(\nu)$ is fixed and allows to solve for both the conserved charges:

$$
\begin{aligned}
M & =\frac{1}{16 G}\left(\nu^{2}+3\right)\left(\left(r_{-}+r_{+}\right)-\frac{\sqrt{r_{+} r_{-}\left(\nu^{2}+3\right)}}{\nu}\right) \\
J & =\frac{l}{32 G}\left(\nu^{2}+3\right)\left(\frac{r_{-} r_{+}\left(3+5 \nu^{2}\right)}{2 \nu}-\left(r_{+}+r_{-}\right) \sqrt{\left(3+\nu^{2}\right) r_{+} r_{-}}\right) .
\end{aligned}
$$

An explicit realization in Einstein gravity was discussed in [54] and is reviewed in appendix A. In the formalism of $[54,55]$, the mass $M$ is identified as the conserved quantity associated to the Killing vector $2 \frac{\partial}{\partial t}$, while the angular momentum is identified as the conserved quantity associated to the Killing vector $-\frac{\partial}{\partial \theta}$. This provides a non-trivial check that the masses guessed by thermodynamics are indeed the same as the ones computed directly in an explicit example, which, unfortunately, has either closed time-like curves (for $\nu^{2}<1$ ) or wrong sign Maxwell term and therefore ghosts (for $\nu^{2}>1$ ).

\subsection{Expectations for the asymptotic rate of growth of complexity}

In [11], it has been proposed that the asymptotic rate of increase of complexity should be proportional to the product of temperature times entropy:

$$
\frac{d C}{d \tau} \simeq T S
$$

The main motivation comes from the fact that complexity growth rate is an extensive quantity which should have the dimensions of an energy, and which should vanish for a static object as an extremal BH. Indeed, for the WAdS BH solutions in Einstein gravity, we find

$$
T S=\frac{\left(r_{+}-r_{-}\right)\left(3+\nu^{2}\right)}{16 G} .
$$

In the next section we will find that the growth rate of the volume of the ERB in a WAdS $\mathrm{BH}$ is indeed proportional to $T S$.

The authors of [62] proposed the following bound for the complexity growth rate:

$$
\frac{d C}{d \tau} \lesssim\left[(M-\Omega J-\Phi Q)_{+}-(M-\Omega J-\Phi Q)_{-}\right],
$$

where \pm indicate that the corresponding values of the quantities are computed at the outer and inner horizons. With suitable units for complexity, the bound (2.14) seems to be saturated in several cases. For WAdS BHs, the angular velocities computed on the inner and outer horizons are:

$$
\Omega_{+}=\frac{2}{l\left(2 \nu r_{+}-\sqrt{\left(\nu^{2}+3\right) r_{+} r_{-}}\right)}, \quad \Omega_{-}=\frac{2}{l\left(2 \nu r_{-}-\sqrt{\left(\nu^{2}+3\right) r_{+} r_{-}}\right)} .
$$


If we use the values of mass and angular momentum in eqs. (2.10)-(2.11), we find that

$$
\left(M-\Omega_{+} J\right)-\left(M-\Omega_{-} J\right)=\frac{\left(r_{+}-r_{-}\right)\left(3+\nu^{2}\right)}{16 G}=T S .
$$

For the purpose of the case studied in this paper, the saturation of the bound in eq. (2.14) is equivalent to eq. (2.12).

\subsection{Eddington-Finkelstein coordinates}

The Eddington-Finkelstein (EF) coordinates can be introduced using the light-like geodesics of the metric in eq. (2.1). A system of EF coordinates for the WAdS BH was already introduced in [61]. The coordinates that we introduce here are not the same, but they are still a system of non-singular coordinates at the horizon, defined using infalling lightlike geodesics, that we find convenient for our purposes. We have the following conserved quantities along geodesics:

$$
\begin{aligned}
K & =2 \dot{t}+\left(2 \nu r-\sqrt{r_{+} r_{-}\left(\nu^{2}+3\right)}\right) \dot{\theta}, \\
P & =\left(2 \nu r-\sqrt{r_{+} r_{-}\left(\nu^{2}+3\right)}\right) \dot{t}+\frac{r}{2} \Psi(r),
\end{aligned}
$$

where dots denote derivatives with respect to the geodesic affine parameter. The null geodesics are found by imposing the additional constraint $d s^{2}=0$. Solving the equation of motion and specializing to $K=0$, we get a particular set of geodesics satisfying

$$
\dot{t}=\frac{P\left(2 \nu r-\sqrt{r_{+} r_{-}\left(\nu^{2}+3\right)}\right)}{\left(\nu^{2}+3\right)\left(r-r_{-}\right)\left(r-r_{+}\right)}, \quad \dot{\theta}=-\frac{2 P}{\left(\nu^{2}+3\right)\left(r-r_{-}\right)\left(r-r_{+}\right)}, \quad \dot{r}= \pm P .
$$

These geodesics can be used to introduce EF coordinates which are regular at the horizon.

The infalling geodesics correspond to the choice of sign

$$
\frac{d \theta}{d r}=\frac{2}{\left(\nu^{2}+3\right)\left(r-r_{-}\right)\left(r-r_{+}\right)}, \quad \frac{d t}{d r}=-\frac{\left(2 \nu r-\sqrt{r_{+} r_{-}\left(\nu^{2}+3\right)}\right)}{\left(\nu^{2}+3\right)\left(r-r_{-}\right)\left(r-r_{+}\right)},
$$

and allow to define EF coordinates $\left(u, \theta_{u}\right)$ such that

$$
d u=d t+\frac{2 \nu r-\sqrt{r_{+} r_{-}\left(\nu^{2}+3\right)}}{\left(\nu^{2}+3\right)\left(r-r_{-}\right)\left(r-r_{+}\right)} d r, \quad d \theta_{u}=d \theta-\frac{2}{\left(\nu^{2}+3\right)\left(r-r_{-}\right)\left(r-r_{+}\right)} d r .
$$

The finite expression for the coordinate change is

$$
u=t+r^{*}(r), \quad \theta_{u}=\theta-\frac{2}{\left(\nu^{2}+3\right)\left(r_{+}-r_{-}\right)} \log \left|\frac{r-r_{+}}{r-r_{-}}\right|,
$$

where

$$
r^{*}(r)=\frac{2 \nu r_{+}-\sqrt{r_{+} r_{-}\left(\nu^{2}+3\right)}}{\left(\nu^{2}+3\right)\left(r_{+}-r_{-}\right)} \log \left|r-r_{+}\right|-\frac{2 \nu r_{-}-\sqrt{r_{+} r_{-}\left(\nu^{2}+3\right)}}{\left(\nu^{2}+3\right)\left(r_{+}-r_{-}\right)} \log \left|r-r_{-}\right| .
$$

In terms of these coordinates, the metric becomes

$$
\frac{d s^{2}}{l^{2}}=d u^{2}-d r d \theta_{u}+\left(2 \nu r-\sqrt{r_{+} r_{-}\left(\nu^{2}+3\right)}\right) d u d \theta_{u}+\frac{r}{4} \Psi(r) d \theta_{u}^{2} .
$$




\section{Complexity $=$ volume}

\subsection{Einstein-Rosen Bridge}

Kruskal extension for WAdS BHs was studied in [61]. The Penrose diagrams for WAdS BHs are the same as the ones for asymptotically flat BHs in $3+1$ dimensional spacetime: for the special cases $r_{-}=0$ and $\frac{r_{+}}{r_{-}}=\frac{4 \nu^{2}}{\nu^{2}+3}$, the diagram is the same as the one for the Schwarzschild BH, while for generic $\frac{r_{+}}{r_{-}}$it is identical to the one for the Reissner-Nordström $\mathrm{BH}$ (see figures 7 and 8 of [61]). It is important to emphasize that in the $\nu=1$ case, which is the AdS case, the Penrose diagram is different and is the usual AdS one.

As done in $[8,11]$ for the AdS and the flat cases, we consider an extremal codimension one bulk surface extending between the left and the right side of the Kruskal diagram; we denote the times at the left and right sides as $t_{L}, t_{R}$, respectively. The dual thermofield double state has the following form:

$$
\left|\Psi_{T F D}\right\rangle \propto \sum_{n} e^{-E_{n} \beta / 2-i E_{n}\left(t_{L}+t_{R}\right)}\left|E_{n}\right\rangle_{R}\left|E_{n}\right\rangle_{L}
$$

where $\left|E_{n}\right\rangle_{L, R}$ refer to the energy eigenstates of left and right boundary theories, $\beta$ is the inverse temperature. The usual time translation symmetry in Schwarzschild coordinates corresponds to a forward time translation on the right side and a backward translation on the left one [9], i.e.

$$
t_{L} \rightarrow t_{L}+\Delta t, \quad t_{R} \rightarrow t_{R}-\Delta t
$$

This corresponds to the invariance of the thermofield double state under the evolution described by the Hamiltonian $H=H_{L}-H_{R}$ in the associated couple of entangled WCFTs. If instead we take time running forward on both the copies of the boundaries, we introduce some genuine time dependence in the problem [10] and the volume of the maximal slice will depend on time [11]. We will then consider the symmetric case with equal boundary times

$$
t_{L}=t_{R}=t_{b} / 2 .
$$

\subsection{Non-rotating case}

In this section we will compute the volume of the ERB as a function of time [11]. We first study the non-rotating case, setting $r_{+}=r_{0}$ and $r_{-}=0$ in the metric in the coordinates (2.23). The minimal volume is chosen along the $0 \leq \theta_{u} \leq 2 \pi$ coordinate, and with profile functions $u(\lambda), r(\lambda)$, written in terms of some parameter $\lambda$. The volume integral will run from some $\lambda_{\min }$ to some $\lambda_{\max }$, with associated radii $r_{\min }$ and $r_{\max }$ :

$$
V=2 \cdot 2 \pi \int_{\lambda_{\min }}^{\lambda_{\max }} d \lambda l^{2} \sqrt{\frac{\dot{u}^{2} r}{4}\left[3\left(\nu^{2}-1\right) r+\left(\nu^{2}+3\right) r_{0}\right]-\left(\dot{u} r \nu-\frac{\dot{r}}{2}\right)^{2}}=4 \pi \int d \lambda \mathcal{L}(r, \dot{r}, \dot{u}) .
$$

The factor 2 takes into account the two sides of the Kruskal extension, the $2 \pi$ is the result of the integration in $\theta_{u}$ and the dots denote derivatives with respect to $\lambda$. The radius $r_{\max }$ plays the role of an ultraviolet cutoff; we will take the limit $r_{\max } \rightarrow \infty$ at the end of the 


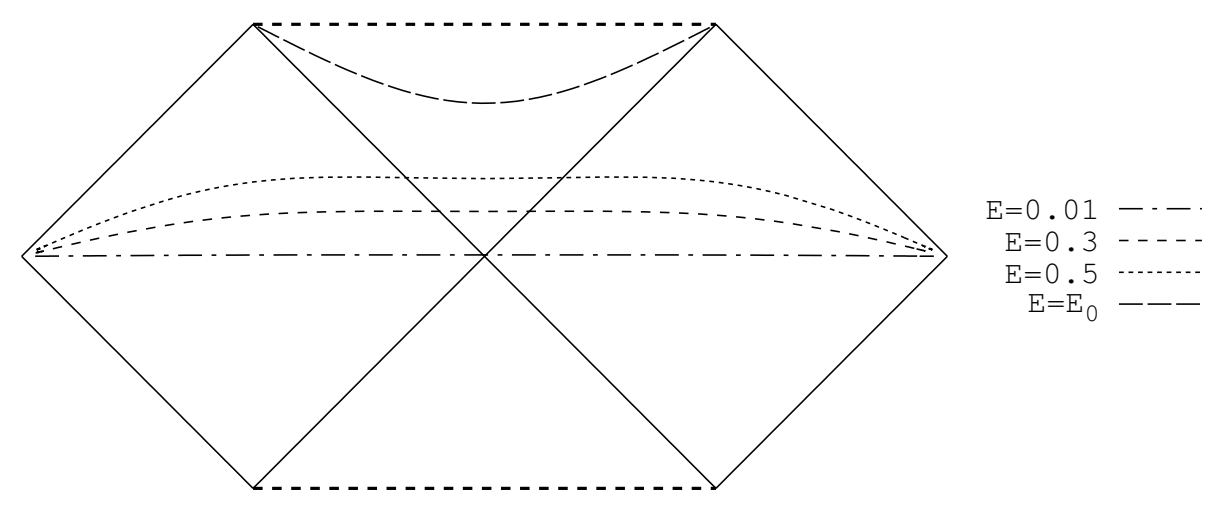

Figure 1. Solutions to eqs. (3.7) for the non-rotating case, plotted in a Penrose diagram, for $\nu=2.5$ and $r_{0}=1$. The $E=E_{0}$ line, which sits at constant $r_{\text {min }}=\frac{r_{0}}{2}$, corresponds to the large $t_{b}$ limit. Penrose diagram coordinates from [61] have been used.

calculation. The conserved quantity from translational invariance in $u$ gives

$$
E=\frac{1}{l^{2}} \frac{\partial \mathcal{L}}{\partial \dot{u}}=\frac{\frac{\nu^{2}+3}{4} \dot{u} r\left(r_{0}-r\right)+\frac{\nu r \dot{r}}{2}}{\sqrt{\frac{\dot{u}^{2} r}{4}\left[3\left(\nu^{2}-1\right) r+\left(\nu^{2}+3\right) r_{0}\right]-\left(\dot{u} r \nu-\frac{\dot{r}}{2}\right)^{2}}} .
$$

It is then useful to gauge the parametrization symmetry for $\lambda$ in such a way that $V=$ $4 \pi l^{2} \int d \lambda$ :

$$
\frac{\dot{u}^{2} r}{4}\left[3\left(\nu^{2}-1\right) r+\left(\nu^{2}+3\right) r_{0}\right]-\left(\dot{u} r \nu-\frac{\dot{r}}{2}\right)^{2}=1, \quad E=\frac{\nu^{2}+3}{4} \dot{u} r\left(r_{0}-r\right)+\frac{\nu r \dot{r}}{2} .
$$

We can then solve for $\dot{r}, \dot{u}$ :

$$
\dot{r}=2 \sqrt{\frac{4 E^{2}+\left(\nu^{2}+3\right) r\left(r-r_{0}\right)}{r\left(3\left(\nu^{2}-1\right) r+\left(\nu^{2}+3\right) r_{0}\right)}}, \quad \dot{u}=\frac{4}{\left(\nu^{2}+3\right)\left(r_{0}-r\right)}\left(\frac{E}{r}-\frac{\nu}{2} \dot{r}\right),
$$

where we took the direction of $\lambda$ in the direction of increasing $r$. These equations can be solved numerically; some example of solutions, plotted in a Penrose diagram, are shown in figure 1.

The minimum radius $r_{\min }$ is a solution of $\dot{r}=0$ :

$$
r_{\text {min }}^{2}-r_{0} r_{\min }+\frac{4 E^{2}}{\left(3+\nu^{2}\right)}=0, \quad r_{\min }=\frac{r_{0}}{2}\left(1 \pm \sqrt{1-\frac{16 E^{2}}{r_{0}^{2}\left(3+\nu^{2}\right)}}\right),
$$

where the physical solution relevant for holographic complexity is the one with the + sign. Conventionally, $t_{b}=0$ corresponds to $E=0$ and $r_{\min }=r_{0}$. The $t_{b} \rightarrow \infty$ limit, instead, corresponds to coincident roots for $r_{\min }$ in eq. (3.8), i.e. $E \rightarrow \frac{r_{0}}{4} \sqrt{\nu^{2}+3}$ and $r_{\min }=\frac{r_{0}}{2}$. The minimal value of the radial coordinate is inside the black hole horizon $\frac{r_{0}}{2} \leq r_{\min } \leq r_{0}$.

The volume can be obtained as an integral in $d r$ :

$$
V=4 \pi l^{2} \int \frac{d r}{\dot{r}}=2 \pi l^{2} \int_{r_{\min }}^{r_{\max }} \sqrt{\frac{r\left(3\left(\nu^{2}-1\right) r+\left(\nu^{2}+3\right) r_{0}\right)}{4 E^{2}+\left(\nu^{2}+3\right) r\left(r-r_{0}\right)}} d r .
$$


The difference of $u$ coordinates is:

$$
\begin{aligned}
& u\left(r_{\max }\right)-u\left(r_{\min }\right)=\int_{r_{\min }}^{r_{\max }} d r \frac{\dot{u}}{\dot{r}} \\
= & \int_{r_{\min }}^{r_{\max }} d r\left[\frac{2}{\left(\nu^{2}+3\right)\left(r_{0}-r\right)}\left(\frac{E}{r} \sqrt{\frac{r\left(3\left(\nu^{2}-1\right) r+\left(\nu^{2}+3\right) r_{0}\right)}{4 E^{2}+\left(\nu^{2}+3\right) r\left(r-r_{0}\right)}}-\nu\right)\right] .
\end{aligned}
$$

Note that this integral is not divergent for $r \rightarrow r_{0}$. The volume can then be written as follows:

$$
\begin{aligned}
\frac{V}{4 \pi l^{2}}= & E\left(u\left(r_{\max }\right)-u\left(r_{\min }\right)\right)+\int_{r_{\min }}^{r_{\max }} d r\left\{\frac{2 \nu E}{\left(\nu^{2}+3\right)\left(r_{0}-r\right)}\right. \\
& \left.-\frac{\sqrt{r\left[4 E^{2}-r\left(r_{0}-r\right)\left(\nu^{2}+3\right)\right]\left[\left(\nu^{2}+3\right) r_{0}+3 r\left(\nu^{2}-1\right)\right]}}{2\left(\nu^{2}+3\right) r\left(r_{0}-r\right)}\right\} .
\end{aligned}
$$

It is important to emphasize that

$$
\lim _{r_{\max } \rightarrow \infty} u\left(r_{\max }\right)-r^{*}\left(r_{\max }\right)=t_{R},
$$

is finite and can be identified with the time at the right boundary. In the limit $r_{\max } \rightarrow \infty$, we can use the explicit expression

$$
u\left(r_{\max }\right)-u\left(r_{\min }\right)=t_{R}+r^{*}\left(r_{\max }\right)-r^{*}\left(r_{\min }\right),
$$

obtained specializing eq. (2.21):

$$
u\left(r_{\max }\right)=t_{R}+r^{*}\left(r_{\max }\right), \quad u\left(r_{\min }\right)=r^{*}\left(r_{\min }\right),
$$

because $t=0$ at $r=r_{\text {min }}$ by symmetry considerations.

Taking into account that both $E$ and $r_{\min }$ depend on $t_{R}$ (see eq. (3.8) for the relation among $r_{\min }$ and $E$ ), the time derivative of eq. (3.11) gives, after several cancellations among terms:

$$
\frac{1}{2 l} \frac{d V}{d t_{R}}=\frac{d V}{d \tau}=2 \pi l E,
$$

where $\tau=l t_{b}=2 l t_{R}$. At large $\tau, E$ approaches to the constant $E_{0}=\frac{r_{0}}{4} \sqrt{\nu^{2}+3}$. Computing the constant of motion $E$ in eq. (3.5) for the particular value $r=r_{\text {min }}$ shows that $E>0$ for $\tau>0$ (corresponding to $\dot{u}>0$ ) and $E<0$ for $\tau<0$ (corresponding to $\dot{u}<0)$. Numerical calculations with the full time dependence can be obtained by expressing $\tau$ in terms of $E$ using eqs. (3.10)-(3.13), are shown in figure 2. For $\nu=1$ the results in $[11,22]$ are recovered, under the change of variables in eq. (2.4).

\subsection{Rotating case}

We use the metric in the coordinates (2.23); the volume functional is:

$$
V=4 \pi \int_{\lambda_{\min }}^{\lambda_{\max }} d \lambda l^{2} \sqrt{\frac{r \dot{u}^{2}}{4} \Psi-\left(\frac{\dot{u}}{2}\left(2 \nu r-\sqrt{r_{+} r_{-}\left(\nu^{2}+3\right)}\right)-\frac{\dot{r}}{2}\right)^{2}}=4 \pi \int d \lambda \mathcal{L}(r, \dot{r}, \dot{u}) .
$$




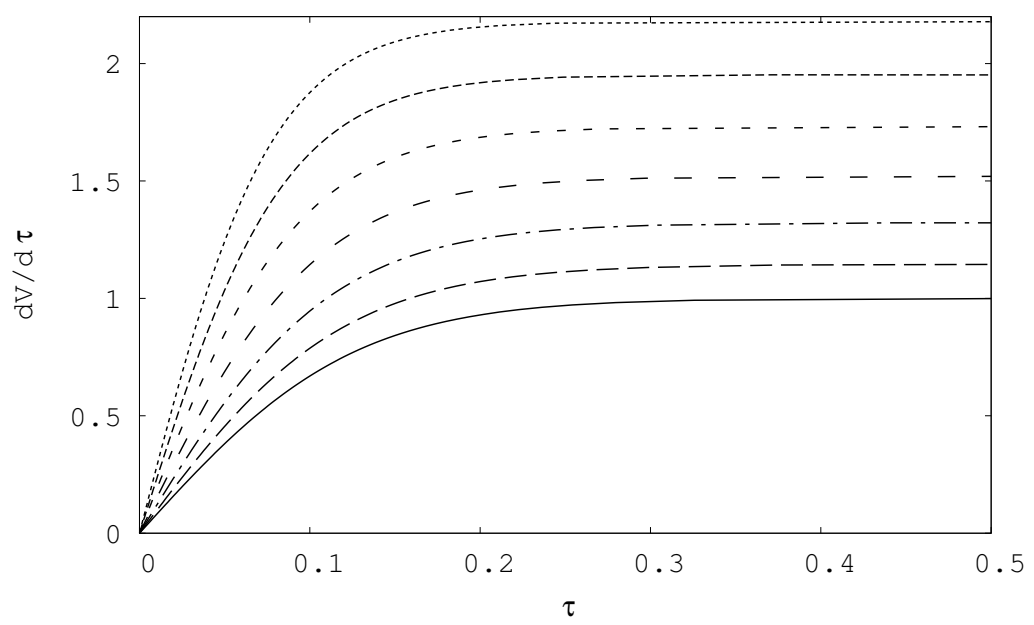

$v=1-$
$v=1.5---$
$v=2---$
$v=2.5--$
$v=3---$
$v=3.5---$

$v=3.5-\cdots--$

Figure 2. Time dependence of $\frac{d V}{d \tau}$ in units of $\pi l$, for $r_{0}=1$ and various values of the warping parameter $\nu$.

Due to the axial symmetry, the volume is taken along the $\theta_{u}$ direction. As before, we find the conserved quantity:

$$
E=\frac{1}{l^{2}} \frac{\partial \mathcal{L}}{\partial \dot{u}}=\frac{\frac{r \dot{u}}{4} \Psi-\left(\frac{\dot{u}}{2}\left(2 \nu r-\sqrt{r_{+} r_{-}\left(\nu^{2}+3\right)}\right)-\frac{\dot{r}}{2}\right) \frac{1}{2}\left(2 \nu r-\sqrt{r_{+} r_{-}\left(\nu^{2}+3\right)}\right)}{\sqrt{\frac{r \dot{u}^{2}}{4} \Psi-\left(\frac{\dot{u}}{2}\left(2 \nu r-\sqrt{r_{+} r_{-}\left(\nu^{2}+3\right)}\right)-\frac{\dot{r}}{2}\right)^{2}}} .
$$

The expression greatly simplifies choosing a parametrization for $\lambda$ such that $V=4 \pi l^{2} \int d \lambda$, which corresponds to setting

$$
\frac{r \dot{u}^{2}}{4} \Psi-\left(\frac{\dot{u}}{2}\left(2 \nu r-\sqrt{r_{+} r_{-}\left(\nu^{2}+3\right)}\right)-\frac{\dot{r}}{2}\right)^{2}=1 .
$$

This gives:

$$
E=-\frac{\nu^{2}+3}{4} \dot{u}\left(r-r_{-}\right)\left(r-r_{+}\right)+\frac{\dot{r}}{4}\left(2 \nu r-\sqrt{r_{+} r_{-}\left(\nu^{2}+3\right)}\right) .
$$

Solving eqs. (3.18), (3.19), we obtain the expressions:

$$
\begin{aligned}
\dot{r}= & 2 \sqrt{\frac{4 E^{2}+\left(\nu^{2}+3\right)\left(r-r_{-}\right)\left(r-r_{+}\right)}{\left(2 \nu r-\sqrt{r_{+} r_{-}\left(\nu^{2}+3\right)}\right)^{2}-\left(\nu^{2}+3\right)\left(r-r_{-}\right)\left(r-r_{+}\right)}}, \\
\dot{u}= & \frac{2}{\left(\nu^{2}+3\right)\left(r-r_{-}\right)\left(r-r_{+}\right)} \\
& {\left[\frac{\sqrt{4 E^{2}+\left(\nu^{2}+3\right)\left(r-r_{-}\right)\left(r-r_{+}\right)}\left(2 \nu r-\sqrt{r_{+} r_{-}\left(\nu^{2}+3\right)}\right)}{\sqrt{\left(2 \nu r-\sqrt{r_{+} r_{-}\left(\nu^{2}+3\right)}\right)^{2}-\left(\nu^{2}+3\right)\left(r-r_{-}\right)\left(r-r_{+}\right)}}-2 E\right] . }
\end{aligned}
$$


The minimum value $r_{\text {min }}$ of the radial coordinate is obtained by solving $\dot{r}=0$ :

$$
r_{\min }=\frac{r_{+}+r_{-}}{2}\left(1 \pm \sqrt{1-\frac{16 E^{2}}{\left(\nu^{2}+3\right)\left(r_{+}+r_{-}\right)^{2}}}\right) .
$$

As in the non-rotating case, the physical solution relevant for holographic complexity is the one with the + sign. Conventionally, $t_{b}=0$ corresponds to $E=0$ and $r_{\min }=r_{+}+r_{-}$. The $t_{b} \rightarrow \infty$ limit corresponds to $E \rightarrow \frac{\left(r_{+}-r_{-}\right)}{4} \sqrt{\nu^{2}+3}$ and $r_{\min }=\frac{r_{+}+r_{-}}{2}$.

The volume can be expressed as an integral in $d r$ as:

$$
V=2 \pi l^{2} \int_{r_{\min }}^{r_{\max }} d r \sqrt{\frac{\left(2 \nu r-\sqrt{r_{+} r_{-}\left(\nu^{2}+3\right)}\right)^{2}-\left(\nu^{2}+3\right)\left(r-r_{-}\right)\left(r-r_{+}\right)}{4 E^{2}+\left(\nu^{2}+3\right)\left(r-r_{-}\right)\left(r-r_{+}\right)}} .
$$

It is useful to introduce the difference among the extremal values of EF coordinates:

$$
\begin{aligned}
& u\left(r_{\max }\right)-u\left(r_{\min }\right)=\int_{r_{\min }}^{r_{\max }} d r \frac{1}{\left(\nu^{2}+3\right)\left(r-r_{-}\right)\left(r-r_{+}\right)}\left[\left(2 \nu r-\sqrt{r_{+} r_{-}\left(\nu^{2}+3\right)}\right)\right. \\
& \left.-2 E \sqrt{\frac{\left(2 \nu r-\sqrt{r_{+} r_{-}\left(\nu^{2}+3\right)}\right)^{2}-\left(\nu^{2}+3\right)\left(r-r_{-}\right)\left(r-r_{+}\right)}{4 E^{2}+\left(\nu^{2}+3\right)\left(r-r_{-}\right)\left(r-r_{+}\right)}}\right] .
\end{aligned}
$$

As in the non-rotating case, $t=0$ at $r=r_{\min }$, and so:

$$
u\left(r_{\max }\right)-u\left(r_{\min }\right)=t_{R}+r^{*}\left(r_{\max }\right)-r^{*}\left(r_{\min }\right) .
$$

By direct computation, we find the relation

$$
\begin{aligned}
\frac{V}{4 \pi l^{2}}= & \int_{r_{\min }}^{r_{\max }} d r\left[\frac{\sqrt{4 E^{2}+\left(\nu^{2}+3\right)\left(r-r_{-}\right)\left(r-r_{+}\right)}}{2\left(\nu^{2}+3\right)\left(r-r_{-}\right)\left(r-r_{+}\right)}\right. \\
& \sqrt{\left(2 \nu r-\sqrt{r_{+} r_{-}\left(\nu^{2}+3\right)}\right)^{2}-\left(\nu^{2}+3\right)\left(r-r_{-}\right)\left(r-r_{+}\right)} \\
& \left.-E \frac{2 \nu r-\sqrt{r_{+} r_{-}\left(\nu^{2}+3\right)}}{\left(\nu^{2}+3\right)\left(r-r_{-}\right)\left(r-r_{+}\right)}\right]+E\left(u\left(r_{\max }\right)-u\left(r_{\min }\right)\right) .
\end{aligned}
$$

Using the previous definitions and simplifying the expression, we obtain again the result

$$
\frac{d V}{d \tau}=2 \pi l E
$$

where $\tau=l t_{b}=2 l t_{R}$. At large $\tau, E$ approaches the constant

$$
E_{0}=\frac{\left(r_{+}-r_{-}\right)}{4} \sqrt{\nu^{2}+3}
$$

Numerical calculation are shown in figure 3. As a consistency check, putting $\nu=1$ for the BTZ case, we find

$$
\lim _{\tau \rightarrow \infty} \frac{d V}{d \tau}=\pi l\left(r_{+}-r_{-}\right)
$$




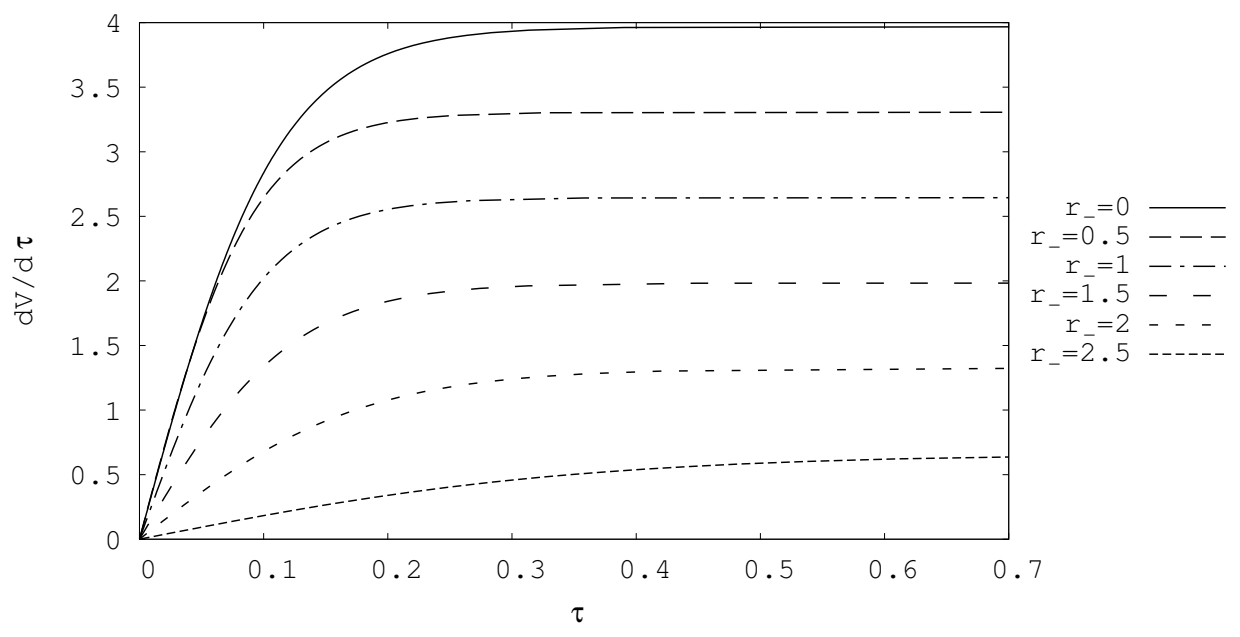

Figure 3. Time dependence of $\frac{d V}{d \tau}$ in units of $\pi l$, for $r_{+}=3, \nu=2$ and several values of $r_{-}$. For other values of $\nu$ the plots are qualitatively similar.

which is the same result found in standard coordinates on the Poincaré patch when we perform the change of variables (2.4).

The late time limit of the maximal volume slices can be found also in a simpler way, as in [11]. In this limit, we expect that the maximal volume slice sits at constant $r$, due to translation invariance in time. We can then consider volume slices at a constant $r=\hat{r}$. Extremizing the volume from the metric in eq. (2.1), we find that the only possible maximal constant- $r$ slice sits at

$$
\hat{r}=\frac{r_{+}+r_{-}}{2} .
$$

Inserting this value back in the volume functional, we recover eq. (3.27) with $E=E_{0}$.

\section{Conclusions}

The result of our calculation gives that the volume of the extremal slices in WAdS is a monotonically growing function for $\tau>0$, whose late time growth rate approaches to

$$
\frac{d V}{d \tau} \rightarrow \frac{\pi l}{2}\left(r_{+}-r_{-}\right) \sqrt{3+\nu^{2}}=S T \frac{8 \pi G l}{\sqrt{3+\nu^{2}}}
$$

where $S$ is the Bekenstein-Hawking entropy and $T$ the Hawking temperature. The late time rate vanishes for extremal black holes $\left(r_{+}=r_{-}\right)$and is proportional to TS.

In $\operatorname{AdS}_{D}$, we have that the coefficient of proportionality between complexity and volume [8] is usually taken as:

$$
C=(D-1) \frac{V}{G l} .
$$

The late-time rate of growth of the volume is:

$$
\lim _{\tau \rightarrow \infty} \frac{d V}{d \tau}=\frac{8 \pi G l}{D-1} S T .
$$


For comparison, in the case of flat spacetime BHs,

$$
\lim _{\tau \rightarrow \infty} \frac{d V}{d \tau} \approx \frac{G r_{h}}{D-3} S T
$$

where $r_{h}$ is the horizon radius ( $\approx$ refer to a neglected order one prefactor [8]). Consequently, the proportionality coefficient between the late time rate of growth of the volume and TS depends on the kind of asymptotic of the spacetime.

In order to compare with the $\mathrm{AdS}_{3}$ case, we can write the rate of growth of the volume in WAdS as:

$$
\frac{d V}{d \tau} \rightarrow S T 4 \pi G l \eta, \quad \eta=\frac{2}{\sqrt{3+\nu^{2}}} .
$$

We may interpret the details of this result in distinct ways, depending on the exact holographic dictionary that we may conjecture between volume and complexity. For example, it could be that complexity approaches at late time to $\eta T S$ (note that $\eta \leq 1$ if we impose $\nu^{2} \geq 1$ ); if this is true, warping would make complexity rate decreases. On the other hand, it could also be that in spaces with WAdS asymptotic the holographic dictionary between complexity and volume is changed by some non-trivial function of the warping parameter $\nu$; for example, if we would have that

$$
C=\frac{2}{G l \eta} V
$$

the asymptotic complexity increase rate would be still $T S$ for every $\nu$. It would be interesting to find arguments in order to be able to discriminate among these various possibilities.

From general considerations based on limits about the speed of computation, there is a conjectured bound on the growth rate of complexity of a physical system [21]. This is called Lloyd's bound and states that

$$
\frac{d C}{d \tau} \leq \frac{2 E}{\pi},
$$

where $E$ is the energy associated to the physical system and units $\hbar=1$ are used. In the case of BHs, the energy is identified with the $\mathrm{BH}$ mass, $E=M$. In general relativity the definition of mass $M$ depends on the choice of the asymptotic Killing vector used to define the conserved quantity. In the WAdS case, the boundary spatial direction $\theta$ in the metric (2.1) is selected by the $r^{2}$ divergence at $r \rightarrow \infty$ in the $d \theta^{2}$ coefficient of the metric. Our definition of mass $M$ in eq. (2.10) is in term of the Killing vector $\xi_{t}=2 \frac{\partial}{\partial t}$; this choice is natural, because the norm of $\xi_{t}$ does not diverge at the boundary. If we would choose another Killing vector to define the mass

$$
\xi_{j}=\xi_{t}-j \frac{\partial}{\partial \theta}
$$

then, for non-zero $j$, the norm of $\xi_{j}$ would diverge as $r^{2}$ for $r \rightarrow \infty$. Instead in the AdS case $(\nu=1)$ the $r^{2}$ divergence of the metric at infinity disappears and $\xi_{t}$ is not a natural time Killing vector, because it is not an eigenvector of the boundary metric. 
Since the late-time complexity rate is proportional to $T S$, if we want that an universal Lloyd's bound holds, we must require that some positive constant $k$ exists such that $k M \geq$ $T S$ for every value of $r_{+}, r_{-}$at fixed $\nu$. If we impose this, we find that $k \geq g(\nu)$, where

$$
g(\nu)=\frac{6 \nu \sqrt{\nu^{2}-1}}{2 \sqrt{3} \nu(2 \nu-n)+3 n \sqrt{\left(\nu^{2}-1\right)}}, \quad n=\sqrt{7 \nu^{2}+4 \sqrt{3} \sqrt{\nu^{2}-1} \nu-3},
$$

which is a decreasing function of $\nu$, with $g(1) \rightarrow \infty$ and $g(\infty) \approx 1.15$. So it is possible, with appropriate normalization, to introduce a Lloyd's bound proportional to $M$ for $\nu>1$. For $\nu=1$ (the AdS case) instead this is not possible; but indeed we know that in this case $M$ is not the correct mass because $\frac{\partial}{\partial t}$ does not correspond to the natural time direction in AdS; in this case there is a conjectured Lloyd's bound in terms of the usual time direction in AdS [13]. For $\nu<1$ we do not expect a Lloyd's bound, because there are closed time-like curves in the geometry; in this range the mass $M$ can even be negative.

Several problems are left for further investigation:

- In this note we considered the case in which WAdS BHs are realized as solutions of Einstein gravity with some appropriate matter content. These objects can also be realized as vacuum solution of TMG and NMG. In these cases we expect some higher order corrections to the CV conjecture, analog to the area corrections in the Wald entropy formula. Some proposals have been discussed in [23, 49]. It would be interesting to compute these corrections explicitly.

- The CA conjecture should also be investigated for the WAdS BH solutions, both in the asymptotic rate of growth and in the initial transient period. This was initiated in [51] for the case of TMG; in this case the late-time complexity rate is not proportional to $T S$, but it still vanishes in the extremal case.

- It would be interesting to study complexity in the boundary Warped CFTs. Lagrangian examples of free Warped CFT were introduced in [36, 37].

\section{Acknowledgments}

We are grateful to Shira Chapman for useful comments on the manuscript.

\section{A An explicit model}

In this appendix we consider an explicit model whose entropy satisfies the area law and admitting the metric eq. (2.1) as a solution [54]. This is a model of Einstein gravity in $(2+1)$ dimensions minimally coupled to a gauge field with Chern-Simons and Maxwell terms:

$$
S=\frac{1}{16 \pi G} \int d^{3} x\left\{\sqrt{g}\left[\left(R+\frac{2}{L^{2}}\right)-\frac{\kappa}{4} F^{\mu \nu} F_{\mu \nu}\right]-\frac{\alpha}{2} \epsilon^{\mu \nu \rho} A_{\mu} F_{\nu \rho}\right\},
$$

where $\epsilon^{\mu \nu \rho}$ is the Levi-Civita tensorial density. Here we put a coefficient $\kappa= \pm 1$ in front of the Maxwell kinetic term. The equations of motion for the gauge field are

$$
D_{\mu} F^{\alpha \mu}=-\frac{\alpha}{\kappa} \frac{\epsilon^{\alpha \nu \rho}}{\sqrt{g}} F_{\nu \rho}
$$


while the Einstein equations are

$$
G_{\mu \nu}-\frac{1}{L^{2}} g_{\mu \nu}=\frac{\kappa}{2} T_{\mu \nu}, \quad T_{\mu \nu}=F_{\mu \alpha} F_{\nu}{ }^{\alpha}-\frac{1}{4} g_{\mu \nu} F^{\alpha \beta} F_{\alpha \beta} .
$$

We consider the set of coordinates $(r, t, \theta)$ where the metric assumes the form (2.1), and we choose a gauge motivated by the ansatz from [54]:

$$
A=a d t+(b+c r) d \theta, \quad F=c d r \wedge d \theta,
$$

where $\{a, b, c\}$ is a set of constants.

In this gauge, the Maxwell equations give:

$$
\alpha=\kappa \frac{\nu}{l} .
$$

From the Einstein equations, in order to require absence of closed time-like curves $(\nu>1)$, we have to choose $\kappa=-1$ and the following value of the parameters:

$$
L=l \sqrt{\frac{2}{3-\nu^{2}}}, \quad c=l \sqrt{\frac{3}{2}\left(\nu^{2}-1\right)},
$$

which are simultaneously defined only when $1<\nu^{2}<3$. So there is conflict between absence of closed time-like curves and presence of ghosts $(\kappa=-1)$.

In ref. [54] the conserved charges associated to asympthotic isometries of the black hole have been computed starting from the following form of the metric in the coordinates $(\tilde{t}, \tilde{r}, \tilde{\theta})$ :

$$
d s^{2}=p d \tilde{t}^{2}+\frac{d \tilde{r}^{2}}{h^{2}-p q}+2 h d \tilde{t} d \tilde{\theta}+q d \tilde{\theta}^{2},
$$

with functions given by

$$
p(\tilde{r})=8 G \mu, \quad q(\tilde{r})=-\frac{4 G \mathcal{J}}{\alpha}+2 \tilde{r}-2 \frac{\gamma^{2}}{L^{2}} \tilde{r}^{2}, \quad h(\tilde{r})=-2 \alpha \tilde{r},
$$

and $\mathrm{U}(1)$ gauge field

$$
A=A_{\tilde{t}} d \tilde{t}+A_{\tilde{\theta}} d \tilde{\theta}, \quad A_{\tilde{t}}(\tilde{r})=\frac{\alpha^{2} L^{2}-1}{\gamma \alpha L}+\zeta, \quad A_{\tilde{\theta}}(\tilde{r})=-\frac{4 G}{\alpha} Q+\frac{2 \gamma}{L} \tilde{r},
$$

where

$$
\gamma=\sqrt{\frac{1-\alpha^{2} L^{2}}{8 G \mu}},
$$

and $\zeta$ is a gauge constant.

We can put the metric (2.1) in the form (A.7) by means of the coordinate change:

$$
\tilde{t}=\sqrt{\frac{l^{3}}{\omega}} t, \quad \tilde{r}=r-\frac{\sqrt{r_{+} r_{-}\left(\nu^{2}+3\right)}}{2 \nu}, \quad \tilde{\theta}=\frac{\sqrt{\omega l^{3}}}{2} \theta,
$$

where

$$
\omega=\frac{\nu^{2}+3}{2 \nu l}\left(\nu\left(r_{+}+r_{-}\right)-\sqrt{r_{+} r_{-}\left(\nu^{2}+3\right)}\right) .
$$


The previous set of transformations is such that the gauge field in the coordinates $(t, r, \theta)$ can be written as $A=a d t+(b+c r) d \theta$, motivating the ansatz (A.4).

The quantities $\mu, \mathcal{J}, Q$ appearing in the previous solution are respectively identified with the mass, angular momentum and charge of the black hole. The equations of motion and the change of coordinates do not uniquely fix the charge $Q$, while we identify

$$
\begin{aligned}
& \mu=\frac{\nu^{2}+3}{16 G l^{2}}\left(r_{+}+r_{-}-\frac{\sqrt{r_{+} r_{-}\left(\nu^{2}+3\right)}}{\nu}\right) \\
& \mathcal{J}=\frac{2 \nu\left(r_{+}+r_{-}\right) \sqrt{r_{+} r_{-}\left(\nu^{2}+3\right)}-\left(5 \nu^{2}+3\right) r_{+} r_{-}}{8 G l\left(\nu\left(r_{+}+r_{-}\right)-\sqrt{r_{+} r_{-}\left(\nu^{2}+3\right)}\right)} .
\end{aligned}
$$

As it is pointed out in [54], the set $\{\mu, \mathcal{J}, Q\}$ satisfies the first law of thermodynamics in the form

$$
d \mu=T d S+\Omega d \mathcal{J}+\Phi_{\text {tot }} d Q
$$

where the total electric potential is shown to be $\Phi_{\text {tot }}=0$, thus eliminating the contribution from the charge of the black hole.

This special form of the first law of thermodynamics is a consequence of the choice of the Killing vectors associated to mass and angular momentum in [54], since all the contributions coming from the charge are eliminated.

A direct match with the mass $M$ and angular momentum $J$ coming from the thermodynamic analysis in $(t, r, \theta)$ coordinates gives:

$$
\mu=\frac{M}{l^{2}}, \quad \mathcal{J}=-\frac{4 J}{\omega l^{2}} .
$$

In order to get the conserved charges associated to isometries in $(t, r, \theta)$ coordinates, we need to adjust the normalization conditions:

- The angular range $0 \leq \theta \leq 2 \pi$ corresponds to $0 \leq \tilde{\theta} \leq 2 \pi \frac{\sqrt{\omega l^{3}}}{2}$, so extensive quantities, such as mass, entropy and angular momentum in $(t, r, \theta)$ coordinates get an extra $\frac{\sqrt{\omega l^{3}}}{2}$ factor if we want to preserve the length of the integration along $[0,2 \pi]$.

- Killing vectors are transformed as:

$$
\frac{\partial}{\partial t}=\sqrt{\frac{l^{3}}{\omega}} \frac{\partial}{\partial \tilde{t}}, \quad \frac{\partial}{\partial \theta}=\frac{\sqrt{\omega l^{3}}}{2} \frac{\partial}{\partial \tilde{\theta}} .
$$

- In [54] it is defined $\Omega=-h\left(r_{+}\right) / q\left(r_{+}\right)$, while in eq. (2.10), (2.11) we followed the conventions of [34], where an additional factor of $l$ is put in the denominator both for the angular velocity and the Hawking temperature. Choosing the last normalization amounts to modify $\mu \rightarrow \mu / l$, with the other conserved charges of the black hole unchanged.

Taking into account all these corrections, we get that the mass in $(t, r, \theta)$ coordinates with the Killing $\frac{\partial}{\partial t}$ is $M / 2$ and the angular momentum associated to the Killing $-\frac{\partial}{\partial \theta}$ is $J$. The $1 / 2$ factor in the normalization of the mass is reminiscent of Komar's anomalous factor and it is also pointed out for similar computations in [42]. 
Open Access. This article is distributed under the terms of the Creative Commons Attribution License (CC-BY 4.0), which permits any use, distribution and reproduction in any medium, provided the original author(s) and source are credited.

\section{References}

[1] J.D. Bekenstein, Black holes and entropy, Phys. Rev. D 7 (1973) 2333 [inSPIRE].

[2] J.M. Bardeen, B. Carter and S.W. Hawking, The Four laws of black hole mechanics, Commun. Math. Phys. 31 (1973) 161 [INSPIRE].

[3] A. Strominger and C. Vafa, Microscopic origin of the Bekenstein-Hawking entropy, Phys. Lett. B 379 (1996) 99 [hep-th/9601029] [INSPIRE].

[4] S. Ryu and T. Takayanagi, Holographic derivation of entanglement entropy from AdS/CFT, Phys. Rev. Lett. 96 (2006) 181602 [hep-th/0603001] [INSPIRE].

[5] H. Casini, M. Huerta and R.C. Myers, Towards a derivation of holographic entanglement entropy, JHEP 05 (2011) 036 [arXiv: 1102.0440] [INSPIRE].

[6] A. Lewkowycz and J. Maldacena, Generalized gravitational entropy, JHEP 08 (2013) 090 [arXiv: 1304.4926] [INSPIRE].

[7] L. Susskind, Computational Complexity and Black Hole Horizons, Fortsch. Phys. 64 (2016) 24 [Addendum ibid. 64 (2016) 44] [arXiv:1403.5695] [INSPIRE].

[8] L. Susskind, Entanglement is not enough, Fortsch. Phys. 64 (2016) 49 [arXiv:1411.0690] [INSPIRE].

[9] J.M. Maldacena, Eternal black holes in anti-de Sitter, JHEP 04 (2003) 021 [hep-th/0106112] [INSPIRE].

[10] T. Hartman and J. Maldacena, Time Evolution of Entanglement Entropy from Black Hole Interiors, JHEP 05 (2013) 014 [arXiv:1303.1080] [INSPIRE].

[11] D. Stanford and L. Susskind, Complexity and Shock Wave Geometries, Phys. Rev. D 90 (2014) 126007 [arXiv: 1406 . 2678] [INSPIRE].

[12] A.R. Brown, D.A. Roberts, L. Susskind, B. Swingle and Y. Zhao, Holographic Complexity Equals Bulk Action?, Phys. Rev. Lett. 116 (2016) 191301 [arXiv:1509.07876] [INSPIRE].

[13] A.R. Brown, D.A. Roberts, L. Susskind, B. Swingle and Y. Zhao, Complexity, action and black holes, Phys. Rev. D 93 (2016) 086006 [arXiv:1512.04993] [InSPIRE].

[14] M.A. Nielsen, A geometric approach to quantum circuit lower bounds, Quant. Inf. Comput. 6 (2006) 213 [quant-ph/0502070].

[15] M.R. Dowling and M.A. Nielsen, The geometry of quantum computation, Quant. Inf. Comput. 8 (2007) 861 [quant-ph/0701004].

[16] R. Jefferson and R.C. Myers, Circuit complexity in quantum field theory, JHEP 10 (2017) 107 [arXiv: 1707.08570] [INSPIRE].

[17] S. Chapman, M.P. Heller, H. Marrochio and F. Pastawski, Toward a Definition of Complexity for Quantum Field Theory States, Phys. Rev. Lett. 120 (2018) 121602 [arXiv: 1707.08582] [INSPIRE].

[18] K. Hashimoto, N. Iizuka and S. Sugishita, Time evolution of complexity in Abelian gauge theories, Phys. Rev. D 96 (2017) 126001 [arXiv:1707.03840] [INSPIRE]. 
[19] P. Caputa, N. Kundu, M. Miyaji, T. Takayanagi and K. Watanabe, Liouville Action as Path-Integral Complexity: From Continuous Tensor Networks to AdS/CFT, JHEP 11 (2017) 097 [arXiv: 1706. 07056] [INSPIRE].

[20] B. Swingle, Entanglement Renormalization and Holography, Phys. Rev. D 86 (2012) 065007 [arXiv:0905.1317] [INSPIRE].

[21] S. Lloyd, Ultimate physical limits to computation, Nature 406 (2000) 1047.

[22] D. Carmi, S. Chapman, H. Marrochio, R.C. Myers and S. Sugishita, On the Time Dependence of Holographic Complexity, JHEP 11 (2017) 188 [arXiv:1709.10184] [INSPIRE].

[23] M. Alishahiha, Holographic Complexity, Phys. Rev. D 92 (2015) 126009 [arXiv:1509.06614] [INSPIRE].

[24] O. Ben-Ami and D. Carmi, On Volumes of Subregions in Holography and Complexity, JHEP 11 (2016) 129 [arXiv: 1609.02514] [INSPIRE].

[25] R. Abt, J. Erdmenger, H. Hinrichsen, C.M. Melby-Thompson, R. Meyer, C. Northe et al., Topological Complexity in AdS3/CFT2, arXiv:1710.01327 [INSPIRE].

[26] M. Miyaji, T. Numasawa, N. Shiba, T. Takayanagi and K. Watanabe, Distance between Quantum States and Gauge-Gravity Duality, Phys. Rev. Lett. 115 (2015) 261602 [arXiv: 1507.07555] [INSPIRE].

[27] S. Chapman, H. Marrochio and R.C. Myers, Complexity of Formation in Holography, JHEP 01 (2017) 062 [arXiv:1610.08063] [INSPIRE].

[28] J.L.F. Barbon and E. Rabinovici, Holographic complexity and spacetime singularities, JHEP 01 (2016) 084 [arXiv: 1509.09291] [INSPIRE].

[29] S. Bolognesi, E. Rabinovici and S.R. Roy, On Some Universal Features of the Holographic Quantum Complexity of Bulk Singularities, arXiv:1802.02045 [INSPIRE].

[30] A.P. Reynolds and S.F. Ross, Complexity of the AdS Soliton, Class. Quant. Grav. 35 (2018) 095006 [arXiv: 1712.03732] [INSPIRE].

[31] M. Moosa, Evolution of Complexity Following a Global Quench, JHEP 03 (2018) 031 [arXiv: 1711.02668] [INSPIRE].

[32] M. Moosa, Divergences in the rate of complexification, Phys. Rev. D 97 (2018) 106016 [arXiv: 1712.07137] [INSPIRE].

[33] Y.-S. An and R.-H. Peng, Effect of the dilaton on holographic complexity growth, Phys. Rev. D 97 (2018) 066022 [arXiv:1801.03638] [INSPIRE].

[34] D. Anninos, W. Li, M. Padi, W. Song and A. Strominger, Warped AdS $S_{3}$ Black Holes, JHEP 03 (2009) 130 [arXiv: 0807.3040] [INSPIRE].

[35] S. Detournay, T. Hartman and D.M. Hofman, Warped Conformal Field Theory, Phys. Rev. D 86 (2012) 124018 [arXiv:1210.0539] [INSPIRE].

[36] D.M. Hofman and B. Rollier, Warped Conformal Field Theory as Lower Spin Gravity, Nucl. Phys. B 897 (2015) 1 [arXiv: 1411.0672] [INSPIRE].

[37] K. Jensen, Locality and anomalies in warped conformal field theory, JHEP 12 (2017) 111 [arXiv:1710.11626] [INSPIRE].

[38] D. Anninos, J. Samani and E. Shaghoulian, Warped Entanglement Entropy, JHEP 02 (2014) 118 [arXiv: 1309.2579] [INSPIRE]. 
[39] A. Castro, D.M. Hofman and N. Iqbal, Entanglement Entropy in Warped Conformal Field Theories, JHEP 02 (2016) 033 [arXiv:1511.00707] [INSPIRE].

[40] T. Azeyanagi, S. Detournay and M. Riegler, Warped Black Holes in Lower-Spin Gravity, arXiv:1801.07263 [INSPIRE].

[41] K.A. Moussa, G. Clement and C. Leygnac, The Black holes of topologically massive gravity, Class. Quant. Grav. 20 (2003) L277 [gr-qc/0303042] [INSPIRE].

[42] A. Bouchareb and G. Clement, Black hole mass and angular momentum in topologically massive gravity, Class. Quant. Grav. 24 (2007) 5581 [arXiv:0706.0263] [inSPIRE].

[43] D. Anninos, Hopfing and Puffing Warped Anti-de Sitter Space, JHEP 09 (2009) 075 [arXiv:0809.2433] [INSPIRE].

[44] M. Bañados, C. Teitelboim and J. Zanelli, The Black hole in three-dimensional space-time, Phys. Rev. Lett. 69 (1992) 1849 [hep-th/9204099] [INSPIRE].

[45] M. Bañados, M. Henneaux, C. Teitelboim and J. Zanelli, Geometry of the (2+1) black hole, Phys. Rev. D 48 (1993) 1506 [Erratum ibid. D 88 (2013) 069902] [gr-qc/9302012] [INSPIRE].

[46] G. Clement, Warped AdS $S_{3}$ black holes in new massive gravity, Class. Quant. Grav. 26 (2009) 105015 [arXiv: 0902 .4634] [INSPIRE].

[47] E. Tonni, Warped black holes in 3D general massive gravity, JHEP 08 (2010) 070 [arXiv: 1006.3489] [INSPIRE].

[48] R.M. Wald, Black hole entropy is the Noether charge, Phys. Rev. D 48 (1993) R3427 [gr-qc/9307038] [INSPIRE].

[49] M. Alishahiha, A. Faraji Astaneh, A. Naseh and M.H. Vahidinia, On complexity for $F(R)$ and critical gravity, JHEP 05 (2017) 009 [arXiv:1702.06796] [INSPIRE].

[50] W.-D. Guo, S.-W. Wei, Y.-Y. Li and Y.-X. Liu, Complexity growth rates for AdS black holes in massive gravity and $f(R)$ gravity, Eur. Phys. J. C 77 (2017) 904 [arXiv:1703.10468] [INSPIRE].

[51] M. Ghodrati, Complexity growth in massive gravity theories, the effects of chirality and more, Phys. Rev. D 96 (2017) 106020 [arXiv:1708.07981] [INSPIRE].

[52] M.M. Qaemmaqami, Complexity growth in minimal massive 3D gravity, Phys. Rev. D 97 (2018) 026006 [arXiv: 1709.05894] [InSPIRE].

[53] M. Gurses, Perfect Fluid Sources in 2+1 Dimensions, Class. Quant. Grav. 11 (1994) 2585 [INSPIRE].

[54] M. Bañados, G. Barnich, G. Compere and A. Gomberoff, Three dimensional origin of Godel spacetimes and black holes, Phys. Rev. D 73 (2006) 044006 [hep-th/0512105] [INSPIRE].

[55] G. Barnich and G. Compere, Conserved charges and thermodynamics of the spinning Godel black hole, Phys. Rev. Lett. 95 (2005) 031302 [hep-th/0501102] [INSPIRE].

[56] G. Compere, S. Detournay and M. Romo, Supersymmetric Godel and warped black holes in string theory, Phys. Rev. D 78 (2008) 104030 [arXiv:0808.1912] [InSPIRE].

[57] S. Detournay and M. Guica, Stringy Schrödinger truncations, JHEP 08 (2013) 121 [arXiv:1212.6792] [INSPIRE]. 
[58] P. Karndumri and E.O. Colgáin, 3D Supergravity from wrapped D3-branes, JHEP 10 (2013) 094 [arXiv: 1307.2086] [INSPIRE].

[59] A. Castro and M.J. Rodriguez, Universal properties and the first law of black hole inner mechanics, Phys. Rev. D 86 (2012) 024008 [arXiv:1204.1284] [InSPIRE].

[60] G. Giribet and M. Tsoukalas, Warped-AdS3 black holes with scalar halo, Phys. Rev. D 92 (2015) 064027 [arXiv: 1506.05336] [INSPIRE].

[61] F. Jugeau, G. Moutsopoulos and P. Ritter, From accelerating and Poincaré coordinates to black holes in spacelike warped $A d S_{3}$ and back, Class. Quant. Grav. 28 (2011) 035001 [arXiv: 1007.1961] [INSPIRE].

[62] R.-G. Cai, S.-M. Ruan, S.-J. Wang, R.-Q. Yang and R.-H. Peng, Action growth for AdS black holes, JHEP 09 (2016) 161 [arXiv: 1606.08307] [INSPIRE]. 